\title{
Chagas Disease Screening Using Point-of-Care Testing in an At-Risk Obstetric Population
}

\author{
Lindsey E. Zamora, ${ }^{1 \star}$ Federico Palacio, ${ }^{2}$ Debra S. Kozlowski, ${ }^{3}$ Kamini Doraivelu, ${ }^{3}$ Carolynn M. Dude, ${ }^{3}$ Denise J. Jamieson, ${ }^{3}$ and \\ Lisa B. Haddad ${ }^{3,4}$ \\ ${ }^{1}$ Department of Obstetrics and Gynecology, Vanderbilt University Medical Center, Nashville, Tennessee; ${ }^{2}$ Division of Infectious Diseases, \\ Department of Medicine, University of Cincinnati, Cincinnati, Ohio; ${ }^{3}$ Department of Gynecology and Obstetrics, Emory University School of \\ Medicine, Atlanta, Georgia; ${ }^{4}$ Center for Biomedical Research, Population Council, New York, New York
}

\begin{abstract}
Congenital transmission is the most important mode of transmission of Chagas disease (CD) in nonendemic countries. Identifying CD in reproductive-aged women is essential to reduce the risk of transmitting the disease to their children and offer treatment to women and their children, which could cure the disease. We evaluated the use of point-of-care (POC) testing for CD in postpartum patients. In our patient population, 16.7\% (23/138) tested positive by POC testing, but confirmatory testing was negative for all patients. Among those considered high risk, $30 \%$ declined participation. Our results suggest limited utility of the point-of-care test used in our study and identify an opportunity for improvement to broaden diagnostic testing options. Our study also highlights the need to develop strategies to increase subject participation in future research.
\end{abstract}

Chagas disease (CD) is a parasitic disease caused by the protozoan Trypanosoma cruzi; it affects more than 5.7 million people worldwide, claims 12,000 lives annually, and causes the greatest burden of disability-adjusted life years of any parasitic disease in Latin America. Approximately 1.2 million of those affected are women of childbearing age. ${ }^{1,2}$ Chagas disease is present in rural areas in endemic countries, and due to migration of infected individuals from rural to urban areas in Latin America and international migration from endemic to non-endemic countries, CD has emerged as a public health concern in non-endemic countries, including the United States. ${ }^{3,4}$ Approximately 23 million people living in the United States were born in CD-endemic countries, and an estimated $>300,000$ people living in the United States are infected with CD. ${ }^{4}$ Most individuals infected are asymptomatic and unaware, making $C D$ challenging to diagnose and treat. For patients with chronic, untreated CD, 20-30\% will ultimately progress to the "determinate" disease form, characterized by irreversible heart and/or gastrointestinal disease with high morbidity and mortality. Diagnosis in the asymptomatic phase is essential to avoid long-term consequences.

In endemic countries, most of the transmission is vector borne; however, $22 \%$ of new Chagas diagnoses are from congenital transmission. ${ }^{2}$ In non-endemic countries, congenital transmission is most common, and therefore the most important mode of transmission. ${ }^{5}$ In addition, mothers who do not know they were infected congenitally can pass the disease to their children. ${ }^{5}$ It is estimated that 40,000 reproductiveaged women are infected with $T$. cruzi in the United States. ${ }^{6}$ Women with CD transmit the disease to their babies during pregnancy at a rate of $1-5 \%$, resulting in congenital Chagas. ${ }^{7,8}$ These rates are based on screening based on maternal history in research studies and are likely slightly underestimated. In public health settings, screening strategies can be based on testing infants based on maternal history or on testing infants based on symptoms, the latter being even more likely to miss cases, as babies with congenital CD are often asymptomatic. In addition, some neonatal screening

\footnotetext{
${ }^{*}$ Address correspondence to Lindsey E. Zamora, Department of Obstetrics and Gynecology, Vanderbilt University Medical Center, 719 Thompson Lane, Suite 27100, Nashville, TN 37204. E-mail:
} lindsey.zamora@vumc.org tests have low sensitivity or limited availability in resourcelimited settings, and many patients do not follow-up for completion of testing. ${ }^{9}$ Symptomatic infants can have low birth weight, anemia, hepatosplenomegaly, or severe infections that can lead to death. Infants with congenital CD who survive the acute phase are presumed to have the same lifetime risk of the "determinate" form (20-30\%) as adults with CD. ${ }^{8,10}$ They also risk transmitting the disease through blood or organ donation or to future children, sustaining the infection across generations in the absence of the vector. Diagnosing a mother or infant with congenital Chagas increases the likelihood that other family members will be diagnosed and offered early treatment. ${ }^{11}$

Treatment is recommended for reproductive-aged women in the absence of Chagas cardiomyopathy and children younger than 18 years. Benznidazole and nifurtimox are the antitrypanosomal drugs used to treat CD. Benznidazole and nifurtimox are approved by the Food and Drug Administration (FDA) for use in children aged 2-12 years and those younger than 18 years, respectively. Both are available off-label for treatment in adults through their distributors. ${ }^{6,12,13}$ Women with CD should not be treated during pregnancy or while breastfeeding because of concerns about medication safety; treatment is recommended after completion of breastfeeding. ${ }^{11,14}$ Treatment in reproductive-aged women can likely decrease the risk of disease transmission to future children. ${ }^{15}$ Infants infected congenitally can achieve a $>90 \%$ cure if treated within the first year of life. ${ }^{16,17}$

Screening for CD in the United States in at-risk reproductiveaged women is currently recommended by the CDC but is not routinely performed because of lack of physician and patient knowledge and dearth of healthcare resources in the at-risk population. ${ }^{11,18,19}$ There are insufficient data in the United States regarding the prevalence of $C D$; thus, the public health burden remains unclear.

This study sought to examine the feasibility of screening for $\mathrm{CD}$ in an at-risk obstetric population in the United States through development of a screening algorithm using point-ofcare (POC) testing and to identify the prevalence of CD in our hospital. Our study was conducted from January to November 2019, at Grady Health System in Atlanta, GA, among postpartum women. The study was approved by Emory School of Medicine and Grady Health System's Institutional Review 
Boards and was funded by the Emory Medical Care Foundation. We chose to approach postpartum patients as, although women should not be treated for CD while pregnant or breastfeeding, many women only receive health care during pregnancy. Thus, this provides a unique window for healthcare access for women and their families, especially given the higher rate of successful treatment among younger children. Our projected sample size at the start of the study was 456 patients based on the desired precision of a $95 \% \mathrm{Cl}$ using an estimated $1.2 \% \pm 1 \%$ prevalence of Chagas from previous studies. ${ }^{20}$ Approximately 3,100 deliveries occur in our hospital annually, of which $20 \%$ meet eligibility requirements; thus, we felt we could meet our projected sample size in 1 year of testing.

Study staff approached postpartum patients, and those who met the inclusion criteria (they or their mothers were born in Mexico, or Central or South America or had a family history of CD) were invited to participate in the study using a standardized study script. Inclusion criteria were chosen to select women with the highest probability of risk for $\mathrm{CD}$. Those who agreed to participate and consented were screened for CD and completed a questionnaire to collect demographic data and information on risk factors including amount of time lived outside the United States, which countries, and characteristics of their housing while living outside the United States.

Diagnosis of CD requires two different format assays with different antigen preparations to be positive. ${ }^{11,21}$ We used POC IgG technology (InBios Chagas Detect Plus, InBios International, Inc., Seattle, WA) as the first step of the two-step confirmation to improve diagnosis because testing could be interpreted at bedside and confirmatory testing drawn in the same clinical episode. This is the first POC IgG serology test, cleared by the FDA for use in 2016, with a reported > 95\% sensitivity and specificity in endemic populations with a high prevalence of CD and $>95 \%$ sensitivity but a lower specificity (88-92\%) in non-endemic populations as shown in a recent study comparing $C D$ testing modalities in U.S. blood donors. ${ }^{21-23}$ Research staff were trained on correct POC test administration per manufacturer instructions and were directly observed in the first week of the study. If a patient had a positive POC test, a venous sample was drawn and sent to Quest laboratories for confirmation via Hemagen ELISA testing for antibodies to $T$. cruzi. This was the first study in the United States examining POC testing for CD screening in pregnancy.

Once initiated, we noted several patients had positive POC tests and negative Quest confirmatory test results. Most of our positive POC tests were faint positives; thus, we contacted InBios to review our test results including photographs of our positive tests, for guidance. The recommendations from InBios were to report even faintly positive tests as positive. Given these recommendations, we consulted with Chagas experts at the CDC, and chose to modify the study protocol to conduct two confirmatory tests, CDC and Quest, after a positive POC test. The CDC uses serology to perform two tests, a recombinant antigen ELISA, Weiner Chagatest ELISA recombinant 3.0, and trypomastigote-excreted secreted antigen immunoblot. If these results are discordant, an immunofluorescent antibody assay is used as a "tie breaker."

We intended to continue our study for 12 months, but because of unanticipated lack of accuracy of the POC test, we stopped our study after the 11 month of enrollment. At study completion, 1,255 patients were approached, and of these, 196 were found to be eligible based on risk factors. Patients were ineligible largely because of not being born in or having lived in an endemic country. One hundred thirty-eight of the eligible patients $(70 \%)$ consented for testing and were enrolled in the study. The demographic characteristics of these patients are shown in Table 1. Among our study cohort, 23 (16.7\%) had positive POC tests; Quest and CDC confirmatory testing was completed for 21 and 18 patients, respectively. All Quest and CDC confirmatory testing were negative. These results are shown in Figure 1. Confirmatory testing was not completed for two patients because the hospital laboratory lost the blood samples drawn following positive POC testing. Because we had no positive confirmatory tests among our study cohort and did not conduct confirmatory tests for

TABLE 1

Demographic and socioeconomic profile of postpartum women in the study population

\begin{tabular}{|c|c|}
\hline Demographics & $\begin{array}{l}\text { Total study population } \\
\quad(N=138), n(\%)\end{array}$ \\
\hline \multicolumn{2}{|l|}{ Age (years) } \\
\hline$<21$ & $19(13.8)$ \\
\hline $21-34$ & 79 (57.3) \\
\hline$>34$ & $40(29.0)$ \\
\hline Born in Latin America & $124(89.9)$ \\
\hline \multicolumn{2}{|l|}{ Birth country } \\
\hline Colombia & $2(1.5)$ \\
\hline El Salvador & $5(3.7)$ \\
\hline Guatemala & $29(21.2)$ \\
\hline Honduras & $18(13.1)$ \\
\hline Mexico & $64(46.7)$ \\
\hline Peru & $2(1.5)$ \\
\hline United States & $14(10.2)$ \\
\hline Venezuela & $3(2.2)$ \\
\hline \multicolumn{2}{|l|}{ Years lived in Latin America } \\
\hline 0 & $4(3.0)$ \\
\hline $1-5$ & $9(6.5)$ \\
\hline $6-10$ & $13(9.4)$ \\
\hline $11-15$ & $9(6.5)$ \\
\hline $16-20$ & $53(38.4)$ \\
\hline $21-25$ & $27(19.6)$ \\
\hline $26-30$ & $17(12.3)$ \\
\hline $31-35$ & $6(4.4)$ \\
\hline \multicolumn{2}{|l|}{ Years lived in the United States } \\
\hline 0 & $6(4.4)$ \\
\hline $1-5$ & $36(26.1)$ \\
\hline $6-10$ & $16(11.6)$ \\
\hline $11-15$ & $28(20.3)$ \\
\hline $16-20$ & $42(30.4)$ \\
\hline $21-25$ & $10(7.3)$ \\
\hline \multicolumn{2}{|l|}{ Education level } \\
\hline Less than high school & 73 (52.9) \\
\hline High school graduate & $50(36.2)$ \\
\hline Some college, no degree & $10(7.3)$ \\
\hline Associate's degree & $1(0.7)$ \\
\hline Bachelor's degree & $2(1.5)$ \\
\hline Graduate or professional degree & $2(1.5)$ \\
\hline \multicolumn{2}{|l|}{ Marital status } \\
\hline Married & $44(31.9)$ \\
\hline Single & $94(68.1)$ \\
\hline \multicolumn{2}{|l|}{ Employment status } \\
\hline Full time & $5(3.6)$ \\
\hline Part time & $6(4.4)$ \\
\hline Unemployed & $127(92.0)$ \\
\hline \multicolumn{2}{|l|}{ Insurance status } \\
\hline Medicaid & $75(54.4)$ \\
\hline Private insurance & $4(3.0)$ \\
\hline Medicare & $34(24.6)$ \\
\hline Uninsured & $25(18.1)$ \\
\hline Lived in rural Latin America $>6$ months & $115(83.3)$ \\
\hline \multicolumn{2}{|l|}{ Prior knowledge of Chagas disease } \\
\hline Yes & $28(20.3)$ \\
\hline No & $110(79.7)$ \\
\hline
\end{tabular}




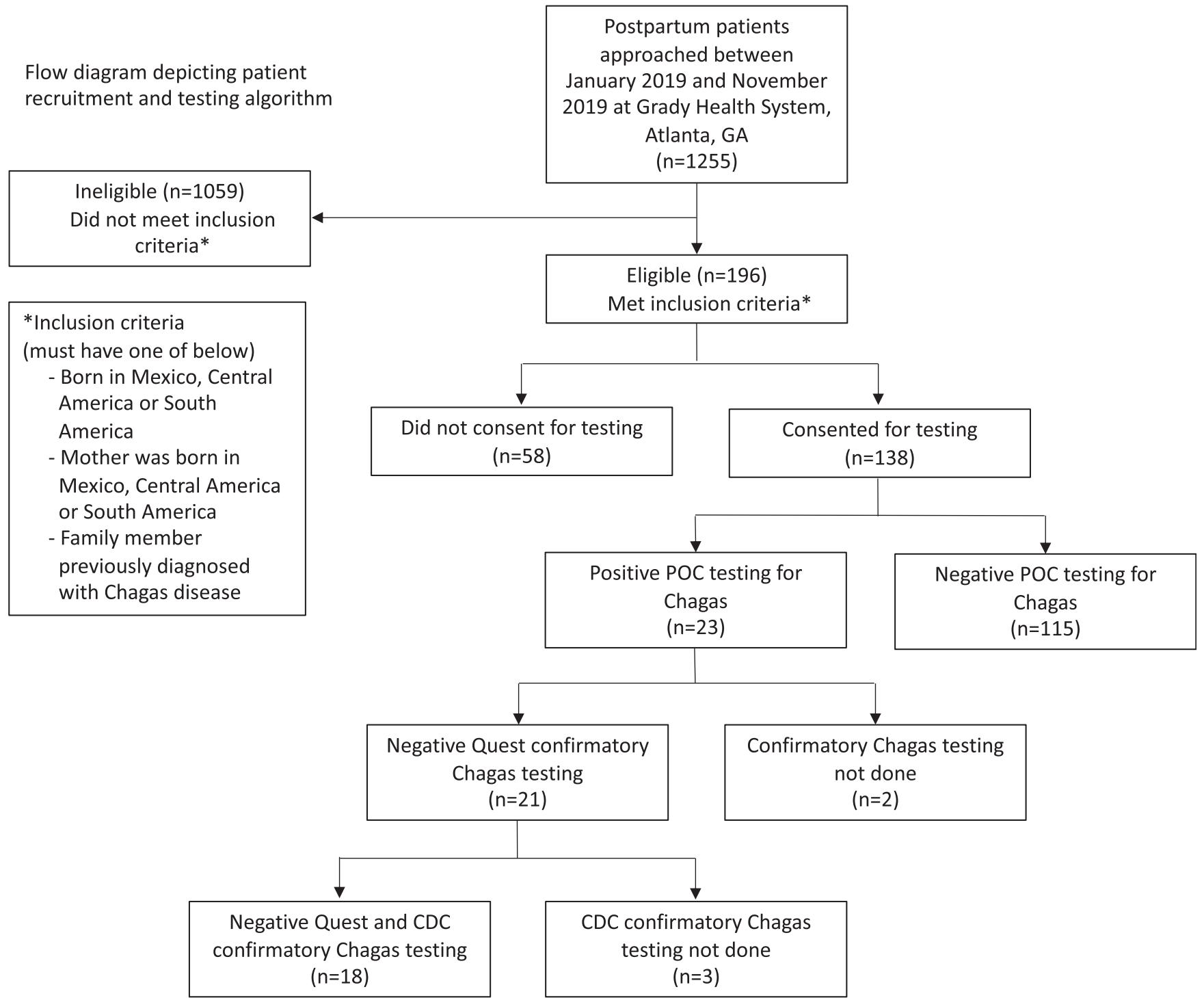

FIGURE 1. Flow diagram depicting patient recruitment and testing algorithm.

patients with negative POC tests, we are not able to calculate diagnostic accuracy measures in our population. Comparing patients with positive and negative POC testing, there were no significant differences in risk factors between the two groups.

We encountered multiple challenges while implementing this study. First, an unexpectedly high number (30\%) of patients who were eligible for the study did not consent to participate, contrary to our anticipated consent rate of $90 \%$. We hired native Spanish speakers as our research staff and purposely did not include any questions relating to patients' legal status in an effort to make patients feel more comfortable during what we anticipated to be a vulnerable time. Research staff also provided basic patient education about CD while conducting the survey in an attempt to spread knowledge and awareness of the disease. Lower rates of consent could be attributed to a number of factors, including stigma surrounding a disease that disproportionally affects the poor and the current political climate surrounding immigrant populations. Unfortunately, reasons for refusal were not collected, and without this information, it is difficult to tell if systematic bias was introduced into the study. This was a missed opportunity that could have given important information leading to improved study design in the future. We feel that making efforts to integrate screening programs into routine health screenings, for example, into yearly well woman examinations or health fairs, could help to destigmatize $C D$ testing and improve consent rates as compared with testing in a research setting. Utilization of community health workers to provide enhanced patient education at the time of testing is another strategy that could be beneficial.

After the first few weeks of testing, the protocol was modified to perform two forms of confirmatory testing. Although patients with positive $\mathrm{POC}$ testing before this protocol change were contacted to return for a second confirmatory test, this proved to be challenging and often unsuccessful. We also attempted to contact patients with lost samples, but were unsuccessful and ultimately did not have confirmatory results for two patients. The patient population at risk for CD in the United States has baseline risk factors for difficulty obtaining healthcare services, and we believe this impacted their ability 
to follow-up. ${ }^{24}$ Although previous studies reported high sensitivity and specificity of this POC test (approximately 95\%) in endemic areas, our results did not echo these findings. We anticipated having a low prevalence of CD in our population, and although the prevalence should not affect the test's specificity, reported specificity for the test has been variable between non-endemic and endemic populations. ${ }^{23}$ Most of our positive POC tests were weakly positive, and although the test's manufacturer advised to report these as positive, reading rapid tests is subjective, and this subjectivity particularly in the case of weak positive results could have impacted our results. Specificity of the POC test based on our study was 83\%, 95\% Cl: 77.1-89.6 (115/138) if Quest and/or CDC testing is treated as the gold standard, and the two specimens with no confirmatory testing were assumed to be negative. Although this specificity estimate is lower than estimates in previous studies that examine this POC test in U.S. populations, it does overlap with the $95 \% \mathrm{Cls}^{23}$ Future study design needs to focus on making data-based sample size calculations to ensure that enough patients are tested to capture true positives. We used an estimated prevalence rate of $1.2 \%$ for our sample size calculation, but other studies in U.S. populations have shown lower prevalence of Chagas. ${ }^{25}$ Given the number of false positives with no true positives obtained, we concluded that there is a need for development of POC tests for CD with higher specificity to use POC testing as a screening tool in our population.

Although we set out to demonstrate the success of an inhospital screening algorithm for CD, our challenges demonstrate the limitations of our current approach. Our research highlights the need for improved availability and quality of screening tests. In addition, research is needed on factors that impact willingness to participate in screening and how to best reach at-risk populations, perhaps by combining screening programs for $\mathrm{CD}$ with screening for more common conditions to maximize screening and benefit to patients. Globally, CD remains an underlying public health challenge, and future research should aim to identify effective strategies in non-endemic countries for capturing patients before disease progression and irreversible health consequences.

Received May 19, 2020. Accepted for publication November 2, 2020.

Published online December 21, 2020.

Financial support: Emory Medical Care Foundation grant funding was used for funding of this study.

Authors' addresses: Lindsey E. Zamora, Department of Obstetrics and Gynecology, Vanderbilt University Medical Center, Nashville, TN, E-mail: lindsey.zamora@vumc.org. Federico Palacio, Division of Infectious Diseases, Department of Medicine, University of Cincinnati, Cincinnati, OH, E-mail: palacifo@ucmail.uc.edu. Debra S. Kozlowski, Kamini Doraivelu, Carolynn M. Dude, Denise J. Jamieson, and Lisa B. Haddad, Department of Gynecology and Obstetrics, Emory University School of Medicine, Atlanta, GA, E-mails: debra.susan.kozlowski@ emory.edu, kamini.doraivelu@emory.edu, carolynn.marie.dude@ emory.edu, djamieson@emory.edu, and lisa.haddad@emory.edu.

This is an open-access article distributed under the terms of the Creative Commons Attribution (CC-BY) License, which permits unrestricted use, distribution, and reproduction in any medium, provided the original author and source are credited.

\section{REFERENCES}

1. Bern C, 2015. Chagas' disease. N Engl J Med 373: 1882.
2. 2015. Chagas disease in Latin America: an epidemiological update based on 2010 estimates. Wkly Epidemiol Rec 90: 33-43.

3. Bern C, Montgomery SP, 2009. An estimate of the burden of Chagas disease in the United States. Clin Infect Dis 49: e52-54.

4. Manne-Goehler J, Umeh CA, Montgomery SP, Wirtz VJ, 2016. Estimating the burden of Chagas disease in the United States. PLoS Negl Trop Dis 10: e0005033.

5. Schenone H, Gaggero M, Sapunar J, Contreras MC, Rojas A, 2001. Congenital Chagas disease of second generation in Santiago, Chile. Report of two cases. Rev Inst Med Trop Sao Paulo 43: 231-232.

6. CDC, Congenital Chagas Disease, Global Health, 2019. Division of Parasitic Diseases. Atlanta, GA: CDC Division of Parasitic Diseases and Malaria. Available at: https://www.cdc.gov/ parasites/chagas/health_professionals/congenital_chagas.html. Accessed February 2, 2020.

7. Howard EJ, Xiong X, Carlier Y, Sosa-Estani S, Buekens P, 2014. Frequency of the congenital transmission of Trypanosoma cruzi: a systematic review and meta-analysis. BJOG 121: 22-33.

8. Edwards MS, Stimpert KK, Bialek SR, Montgomery SP, 2019. Evaluation and management of congenital Chagas disease in the United States. J Pediatr Infect Dis Soc 8: 461-469.

9. Bern C et al., 2009. Congenital Trypanosoma cruzi transmission in Santa Cruz, Bolivia. Clin Infect Dis 49: 1667-1674.

10. Lopez-Velez RNF, Bern C. 2013. American trypanosomiasis (Chagas disease). In: Magill AJRE, Solomon T, Hill DR, eds. Tropical Medicine and Emerging Infectious Disease. 9th edition. Philadelphia, PA: Saunders.

11. CDC, 2012. Congenital transmission of Chagas disease-Virginia, 2010. MMWR Morb Mortal Wkly Rep 61: 477-479.

12. Pan American Health Organization, 2019. Guidelines for the Diagnosis and Treatment of Chagas Disease. Washington, DC: PAHO. Available at: http://iris.paho.org/xmlui/bitstream/handle/123456789/ 49653/9789275120439. Accessed December 10, 2019.

13. U.S. Food and Drug Administration, 2020. Approves Lampit $\circledast$ (Nifurtimox) for the Treatment of Chagas Disease in Children. Press release. Whippany, NJ: Business Wire. Available at: https://www.businesswire.com/news/home/20200807005073/ en/U.S.-Food-and-Drug-Administration-Approves-Lampit\%C2\% AE-nifurtimox-for-the-Treatment-of-Chagas-Disease-in-Children. Accessed August 30, 2020.

14. Bern C, Martin DL, Gilman RH, 2011. Acute and congenital Chagas disease. Adv Parasitol 75: 19-47.

15. Fabbro DL, Danesi E, Olivera V, Codebó MO, Denner S, Heredia C, Streiger M, Sosa-Estani S, 2014. Trypanocide treatment of women infected with Trypanosoma cruzi and its effect on preventing congenital Chagas. PLoS Negl Trop Dis 8: e3312.

16. Russomando G, de Tomassone MM, de Guillen I, Acosta N, Vera N, Almiron M, Candia N, Calcena MF, Figueredo A, 1998. Treatment of congenital Chagas' disease diagnosed and followed up by the polymerase chain reaction. Am J Trop Med Hyg 59: 487-491.

17. Altcheh J, Biancardi M, Lapena A, Ballering G, Freilij H, 2005. Congenital Chagas disease: experience in the hospital de Ninos, Ricardo Gutierrez, Buenos Aires, Argentina. Rev Soc Bras Med Trop 38 (Suppl 2): 41-45.

18. Hotez PJ, Dumonteil E, Betancourt Cravioto M, Bottazzi ME, Tapia-Conyer R, Meymandi S, Karunakara U, Ribeiro I, Cohen RM, Pecoul B, 2013. An unfolding tragedy of Chagas disease in North America. PLoS Negl Trop Dis 7: e2300.

19. Stillwaggon E, Perez-Zetune V, Bialek SR, Montgomery SP, 2018. Congenital Chagas disease in the United States: cost savings through maternal screening. Am J Trop Med Hyg 98: 1733-1742.

20. Meymandi SK, Forsyth CJ, Soverow J, Hernandez S, Sanchez D, Montgomery SP, Traina M, 2017. Prevalence of Chagas disease in the Latin American-born population of Los Angeles. Clin Infect Dis 64: 1182-1188.

21. Eguez KE, Alonso-Padilla J, Teran C, Chipana Z, García W, Torrico F, Gascon J, Lozano-Beltran DF, Pinazo MJ, 2017. Rapid diagnostic tests duo as alternative to conventional serological assays for conclusive Chagas disease diagnosis. PLoS Negl Trop Dis 11: e0005501. 
22. Shah V et al., 2014. Field evaluation of the InBios Chagas detect plus rapid test in serum and whole-blood specimens in Bolivia. Clin Vaccin Immunol 21: 1645-1649.

23. Whitman JD, Bulman CA, Gunderson EL, Irish AM, Townsend RL, Stramer SL, Sakanari JA, Bern C, 2019. Chagas disease serological test performance in U.S. Blood donor specimens. J Clin Microbiol 57: e01217-19.
24. Paz K, Massey KP, 2016. Health disparity among Latina women: comparison with non-Latina women. Clin Med Insights Womens Health 9 (Suppl 1): 71-74.

25. Edwards MS, Rench MA, Todd CW, Czaicki N, Steurer FJ, Bern C, Montgomery SP, 2015. Perinatal screening for Chagas disease in Southern Texas. J Pediatr Infect Dis Soc 4: 67-70. 